\title{
Evaluating Prediction of Continuous Clinical Values: A Glucose Case Study
}

\author{
George Hripcsak ${ }^{1,2}$ David J. Albers ${ }^{1,3}$ \\ ${ }^{1}$ Department of Biomedical Informatics, Columbia University, New \\ York, New York, United States \\ 2 Medical Informatics Services, NewYork-Presbyterian Hospital, New \\ York, New York, United States \\ 3 Department of Pediatrics, University of Colorado Denver-Anschutz \\ Medical Campus, Denver, Colorado, United States \\ Methods Inf Med 2022;61:e35-e44.
}

Address for correspondence George Hripcsak, MD, MS, Department of Biomedical Informatics, Columbia University Medical Center, 622 W 168th Street, PH20, New York, NY 10027, United States (e-mail: hripcsak@columbia.edu).

\begin{abstract}
Background It would be useful to be able to assess the utility of predictive models of continuous values before clinical trials are performed.

Objective The aim of the study is to compare metrics to assess the potential clinical utility of models that produce continuous value forecasts.

Methods We ran a set of data assimilation forecast algorithms on time series of glucose measurements from neurological intensive care unit patients. We evaluated the forecasts using four sets of metrics: glucose root mean square (RMS) error, a set of metrics on a transformed glucose value, the estimated effect on clinical care based on an insulin guideline, and a glucose measurement error grid (Parkes grid). We assessed correlation among the metrics and created a set of factor models.

Results The metrics generally correlated with each other, but those that estimated the effect on clinical care correlated with others the least and were generally associated with their own independent factors. The other metrics appeared to separate into those that emphasized errors in low glucose versus errors in high glucose. The Parkes grid was well correlated with the transformed glucose but not the estimation of clinical care.

Keywords

- metrics

- predictive models

- data assimilation

- machine learning

Discussion Our results indicate that we need to be careful before we assume that commonly used metrics like RMS error in raw glucose or even metrics like the Parkes grid that are designed to measure importance of differences will correlate well with actual effect on clinical care processes. A combination of metrics appeared to explain the most variance between cases. As prediction algorithms move into practice, it will be important to measure actual effects.
\end{abstract}

\section{Introduction}

Using data assimilation and machine learning ${ }^{1,2}$ combined with physiological glucose-insulin models, ${ }^{3,4}$ we have built a glucose prediction system ${ }^{5}$ that is tuned to a patient's own physiology. The glucose-insulin system is represented as a set of equations with physiological parameters that move relatively slowly over time like insulin resistance and patient states that move quickly in time like glucose level. We wish to compare different versions of the system for likely utility in received

June 18, 2021

accepted after revision

December 28, 2021

published online

February 23, 2022
DOI https://doi.org/

$10.1055 / \mathrm{s}-0042-1743170$.

ISSN 0026-1270.

\footnotetext{
(c) 2022. The Author(s).

This is an open access article published by Thieme under the terms of the Creative Commons Attribution-NonDerivative-NonCommercial-License, permitting copying and reproduction so long as the original work is given appropriate credit. Contents may not be used for commercial purposes, or adapted, remixed, transformed or built upon. (https://creativecommons.org/ licenses/by-nc-nd/4.0/) Georg Thieme Verlag KG, Rüdigerstraße 14, 70469 Stuttgart, Germany
} 
aiding glucose therapy decisions. Pending actual development of a decision aid and deployment into clinical practice with a clinical trial, we seek metrics that are likely to reflect utility. Generic summary measures like root mean square (RMS) error are often used for continuous measures like glucose but do not necessarily capture clinical utility. For example, the clinical significance of the difference between blood glucose of $40 \mathrm{mg} / \mathrm{dL}$ and $60 \mathrm{mg} / \mathrm{dL}$ is much larger than between $240 \mathrm{mg} / \mathrm{dL}$ and $260 \mathrm{mg} / \mathrm{dL}$; capturing this requires knowledge of physiology. Furthermore, commonly used summary measures often assume simple statistical distributions that may not be appropriate for medicine. ${ }^{6}$

In this study, we enumerate several possible metrics for assessing goodness of the glucose predictions, pulling from several sources like clinical practice guidelines. In the absence of the gold standard (i.e., a clinical trial of actual utility), we run our prediction system on data from patients in our initial clinical area, the neurological intensive care unit, and we review the correlations among them. We report our approach in the hope that it can be extended to other biomedical areas.

\section{Methods}

\section{Predictive Algorithm}

We tested several evaluation metrics in the context of a predictive algorithm ${ }^{5}$ that uses one data assimilation method, an ensemble Kalman filter, ${ }^{7}$ paired with four mechanistic glucose-insulin models. This paper is not focused on evaluation of the specific data assimilation methods or physiologic models or their pairings but rather on an evaluation methodology for data assimilation-based forecasting methodology within the context of clinical biomedicine, and in particular, within the context of potential application of physiological forecasting within the context of clinical decision support. We therefore primarily describe the evaluation metrics in detail and provide a summary of the data assimilation methods and physiologic models with links to further detail.

Data assimilation ${ }^{1,2}$ is a technique that optimizes the parameters of a mechanistic model by applying the model to a current physical state to make a prediction and then adjusts those parameters based on the difference between the prediction and the actual subsequent state. In this study, the model parameters can include insulin sensitivity, pancreatic $\beta$ cell mass, and liver glucose production, the patient physical state can include the current glucose and insulin levels, and the model is a set of ordinary differential equations that predict the rate of change of the state based on the current state and the model parameters. If predicted glucose differs from the subsequent glucose that is actually observed, data assimilation adjusts the model parameters to tend toward better prediction. The corrections are applied iteratively and over time, and the parameters should move to optimal levels for that patient. We used four glucose models that varied in complexity from a simple exponential decay to two ultradian models of glucose-insulin physiology to a model that also included meal mechanics. In the first model, for any measured glucose away from the mean for that patient, the model has glucose decay exponentially toward the mean value. The ultradian models ${ }^{3}$ represent glucose production and utilization and insulin secretion and elimination using up to six state variables and 30 parameters such that 100 to 150 minute glucose oscillations are properly modeled. The two ultradian versions differ in that the "long" one uses all the states and parameters and the "short" one uses just a subset. The meal model ${ }^{4}$ explicitly includes nutrition in an expanded model with 12 state variables and 70 parameters.

Because the models are non-linear, we used an ensemble Kalman filter ${ }^{7}$ to determine the corrections to parameters needed at each iteration. It differs from a simple Kalman filter in that instead of calculating the parameter changes, it estimates them using a distribution of data points to which it applies the non-linear model, seeing how it affects the distribution.

We ran each of the four models on nine patients. Some models did not converge for some patients, so nine patients with four models each resulted in 29 successful runs.

\section{Data}

We used glucose measurements from Columbia University Irving Medical Center derived from laboratory values and fingerstick glucose meters in the neurological intensive care unit. Approval was obtained from the Columbia University institutional review board.

Our selection process was as follows. We started with a base population of 852 patients from the neurological intensive care unit. Our initial screening criteria were patients with more than a 4-day intensive care unit length of stay with at least 20 glucose measurements per day, and we excluded patients with type 1 diabetes mellitus because the glucose models we used assume there is insulin production.

From this list, we selected patients at random. We reviewed the list to ensure we included patients both with and without exogenous insulin administration. Because we were focused on glycemic control in average patients under the stress of the neurological intensive care unit, we did not require or exclude type 2 diabetes mellitus, but left it to the random selection; in practice, no patients selected had type 2 diabetes mellitus.

We limited the study to nine patients because of the amount of work required to abstract all glucose measurement, insulin administration, feeding, and glucose infusion information for patients with prolonged lengths of stay. Further, running the multiple algorithms on each patient required manual adjustment of parameters.

The cohort is shown in - Table 1. Individually identifiable information has been removed and numeric values have noise added to them. Comorbidities were recorded but were withheld from the table to further protect patient privacy. Older patients had hypertension, congestive heart failure, or coronary artery disease, and one patient had mild renal insufficiency, but the comorbidities were otherwise uninformative. 
Table 1 Participants ${ }^{a}$

\begin{tabular}{|l|l|l|l|l|}
\hline Participant & Age group & Primary diagnosis & $\begin{array}{l}\text { ICU length } \\
\text { of stay (binned days) }\end{array}$ & $\begin{array}{l}\text { Glucose coefficient } \\
\text { of variation }\end{array}$ \\
\hline 1 & $30-34$ & Subarachnoid hemorrhage & $16-20$ & 0.22 \\
\hline 2 & $60-64$ & Intraventricular hemorrhage & $11-15$ & 0.13 \\
\hline 3 & $60-64$ & Subarachnoid hemorrhage & $16-20$ & 0.23 \\
\hline 4 & $20-24$ & Epidural hematoma from trauma & $5-10$ & 0.19 \\
\hline 5 & $60-65$ & Intracerebral hemorrhage & $11-15$ & 0.22 \\
\hline 6 & $20-24$ & Autoimmune encephalitis & $>20$ & 0.25 \\
\hline 7 & $40-45$ & Subarachnoid hemorrhage & $16-20$ & 0.21 \\
\hline 8 & $30-34$ & Subarachnoid hemorrhage & $5-10$ & 0.22 \\
\hline 9 & $40-44$ & Subarachnoid hemorrhage & $16-20$ & 0.33 \\
\hline
\end{tabular}

${ }^{a}$ We added noise to numeric values to assist in protecting patient privacy.

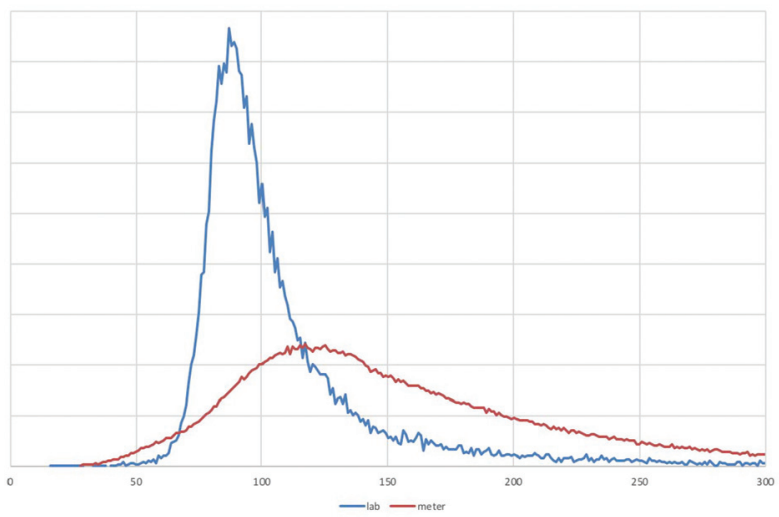

Fig. 1 Distribution of glucose. Distribution of glucose levels in the Columbia University Irving Medical Center Database, showing laboratory values (blue) and portable glucose meter measurements (red).

To better justify our selection of metrics below, we illustrate the challenge with several figures. - Fig. 1 shows the distribution of blood glucose in laboratory tests and in fingerstick glucose meters at our medical center. Meters are higher on average because they tend to be used for patients with glucose intolerance or frank diabetes. Both distributions are clearly non-normal.

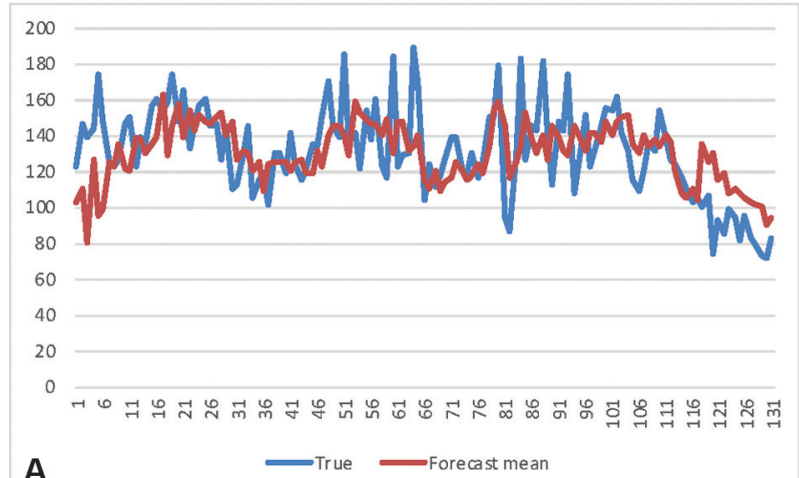

A

—True —orecast mean
-Fig. 2A shows the finger stick glucose of a typical type 2 diabetes patient (we reparametrize to sequence time, simply numbering measurements instead of plotting actual time, based on our previous finding ${ }^{8}$ that stationarity is improved and to simplify comparisons). Superimposed on the figure is a set of glucose predictions for that patient generated by our dataassimilation-based glucose forecaster, ${ }^{5,9}$ which we use for illustration in this paper. In $=$ Fig. $2 A$, the predictions and actual values are well aligned. - Fig. 2B shows a different patient and that patient's predictions; they are clearly misaligned early on, with predictions near zero at one point. The question we address in this paper is how to judge the relative value for predictions like these.

\section{Metrics}

We took four general approaches for metrics (summarized in -Table 2). Our first approach is a simple aggregation of the difference in glucose between the forecast and the measured values. A second approach is to transform glucose to a more clinically relevant scale such that differences anywhere in the scale are approximately linear with impact. A third approach is to assess how the forecast value and the measured value differ in what clinical care would have been given, and therefore what impact the difference might have had. A fourth use is a clinical impact grid intended for glucose meters.

Fig. 2 Glucose time series and predictions for two patients. Time series of glucose levels for two patients, comparing the true levels (blue) to the forecast levels (red). 
Table 2 Metrics

\begin{tabular}{|c|c|}
\hline Short name & Metric \\
\hline \multicolumn{2}{|l|}{ Raw data } \\
\hline RMS & $\begin{array}{l}\text { Root mean square difference in glu- } \\
\text { cose pairs }\end{array}$ \\
\hline \multicolumn{2}{|l|}{ Based on $g$} \\
\hline RMS (g)\% & $\begin{array}{l}100 \times \text { root mean square difference in } g \\
\text { (glucose) pairs }\end{array}$ \\
\hline Max cost $\%$ & $\begin{array}{l}100 \times \max \{\text { (difference in g pairs }) \times \\
(\mathrm{rms} \text { distance in } \mathrm{g} \text { from } 0)\}\end{array}$ \\
\hline Peak max\% & $100 \times($ difference in peak $\mathrm{g}) \times($ peak $\mathrm{g})$ \\
\hline Peak min\% & $\begin{array}{l}100 \times(\text { difference in smallest } \mathrm{g}) \times \\
(\text { smallest } \mathrm{g})\end{array}$ \\
\hline Avg $\times 10,000$ & $\begin{array}{l}10,000 \times(\text { difference in average } \mathrm{g}) \times \\
(\text { larger distance of average } \mathrm{g} \text { from } 0)\end{array}$ \\
\hline \multicolumn{2}{|l|}{ Treatment-based } \\
\hline Insulin & Maximum difference in insulin pairs \\
\hline Bolus & $\begin{array}{l}\text { Maximum difference in glucose bolus } \\
\text { pairs }\end{array}$ \\
\hline Hold & $\begin{array}{l}\text { Difference in whether or not to hold } \\
\text { insulin at any time point }\end{array}$ \\
\hline Notify & $\begin{array}{l}\text { Difference in whether or not to notify } \\
\text { doctor at any time point }\end{array}$ \\
\hline \multicolumn{2}{|l|}{ Parkes-based } \\
\hline Avg Parkes\% & $100 \times$ average Parkes class \\
\hline RMS Parkes\% & $\begin{array}{l}100 \times \text { root mean square Parkes }(A=0, \\
B=1, C=2 D=3, E=4)\end{array}$ \\
\hline Max Parkes & Maximum Parkes class \\
\hline
\end{tabular}

\section{Root Mean Square Difference}

For simple aggregation of the difference in glucose, we estimated the RMS of the simple difference in measured blood glucose level versus forecast level at each time point. This one is the most easily explained and understood and most commonly used. ${ }^{10}$ It will tend to emphasize differences at high glucoses, missing the critical importance of hypoglycemia. The metric is generally used for verification ${ }^{11}$-showing that the model produces accurate forecasts-rather than utility, but we include it as it is a common metric and we wish to see its relation to metrics intended to better assess impact. (We also distinguish verification from validation, the latter testing if the model is acting as we expect, be it accurate or not.)

\section{Transformed Glucose}

We generated a glucose level transformation using the scale in - Table 3 of approximate consequences of having a glucose at that level (set by the authors but also informed by case series $^{12,13}$ ), and assigned a very approximate cost impact changing by a factor of 10 at each level (dollar cost of insurance payout from death, intensive care unit stay, emergency department visit, office visit, change in dose at home). We found empirically that this scale resulted in too high a focus on extreme events, so we switched to a logarithm scale of cost. We then developed a transformation that would map from measured glucose approximately to the logarithm of cost at each level. We also considered logarithm of raw glucose, but rejected it because it overemphasized low glucose with little input about high glucose. The following formula for transformation,

$$
g=0.5-\frac{1}{1+\frac{\text { glucose }}{120}}
$$

maps the glucose range of 0 to infinity to a range of -0.5 to 0.5 , approximating the log cost but signed so that low glucose is negative. Given this transformation, we can calculate several aggregations (i.e., several loss functions): RMS difference in $\mathrm{g}$ (instead of raw glucose) over estimates; the maximum "cost" (not monetary but in terms of total impact), defined as the difference in $\mathrm{g}$ between the forecast and measured value times the distance from the center of the scale, 120; difference in highest $\mathrm{g}$ forecast to highest $\mathrm{g}$

Table 3 Blood glucose transformation

\begin{tabular}{|l|l|l|l|l|}
\hline Glucose $\mathbf{( m g / d L )}$ & Clinical impact & Approximate cost impact & $\mathbf{0 . 1} \times \log (\operatorname{cost})$ & $\mathbf{g}$ \\
\hline 0 & Death & 100,000 & 0.5 & -0.50 \\
\hline 20 & Coma & 10,000 & 0.4 & -0.36 \\
\hline 40 & Obvious symptoms & 1,000 & 0.3 & -0.25 \\
\hline 65 & Symptoms start & 100 & 0.2 & -0.15 \\
\hline 80 & Normal lower & 10 & 0.1 & -0.1 \\
\hline 120 & Center & 1 & 0 & 0 \\
\hline 180 & Target upper for DM & 10 & 0.1 & 0.1 \\
\hline 250 & Symptoms start & 100 & 0.2 & 0.18 \\
\hline 350 & Symptoms obvious & 1,000 & 0.3 & 0.24 \\
\hline 600 & Coma & 10,000 & 0.4 & 0.33 \\
\hline$\infty$ & Death & 100,000 & 0.5 & 0.50 \\
\hline
\end{tabular}




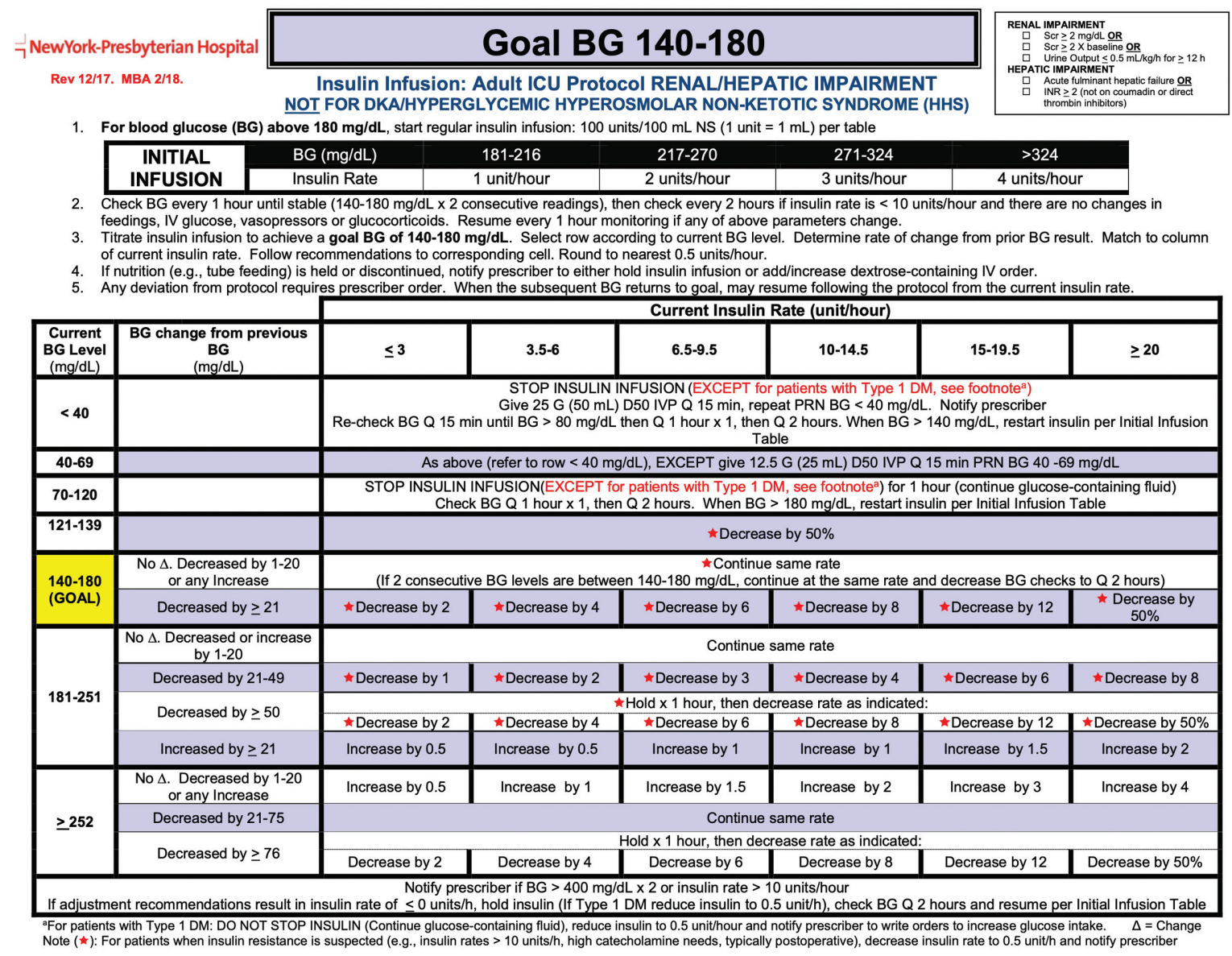

Fig. 3 Insulin administration guideline. This guideline dictates insulin rate and other interventions based on new blood glucose measurements and the history of previous insulin doses. We used this guideline to estimate the effect that a difference in glucose level (actual vs. forecast) might have had on clinical care. (The figure is supplied only for illustration of the glucose algorithm. Any incorporation into practice must be done via appropriate local clinical confirmation and review. Image courtesy: NewYork-Presbyterian Hospital.)

measured to accommodate differences in timing; analogous difference in lowest $g$; and difference in mean $g$ times the larger mean g ("mean cost").

\section{Insulin Administration Guideline}

For changes in clinical care, we used an intensive care unit insulin administration guideline to judge difference between forecast and measurement. The guideline (-Fig. 3) specifies actions like insulin dose and timing and glucose boluses based on measured glucose and current insulin administration. We start the - Fig. 3 algorithm with zero insulin and follow the treatment recommendations for the time series once using the forecast values and a second time using measured values. For example, based on the first glucose measurement, say 220 , we set the initial insulin dose, in this case 2 units per hour. If the next measurement is 190, then we would decrease the rate to 1 unit per hour (because it matches the row for glucose 181 to 251 that is decreased by 21 to 49, which recommends to decrease the rate by 1 unit per hour). We do this both for the measured values and for the forecasted values. We then look at the difference in insulin dose (primary outcome) for the forecast versus measured value, as well as any change in emergency bolus of glucose, change in a hold order on insulin administration, and change in need to notify the physician. We select the largest difference in the time series.

\section{Parkes Error Grid}

We also use the Parkes error grid (-Fig. 4), ${ }^{14,15}$ which was a teaching tool that was adapted for assessing the clinical accuracy of blood glucose meters. For every forecast versus measured pair, the grid assigns one of six categories of impact, denoted $\mathrm{A}$ to $\mathrm{F}$ and rated none too dangerous; we assign a number from 1 to 6 . For this score, we aggregated the average Parkes error zone, the RMS Parkes error zone, and the maximum Parkes error zone, which indicates the highest potential clinical impact.

\section{Evaluation}

For our evaluation, we selected nine representative cases similar to those in -Fig. 2A and B, each with a time series of glucose measurements, and we made predictions using each of four variations of our data assimilation method depending on what physiological model we used. For each set of predictions, we calculated the results of 13 metrics defined above and shown in -Table 2 . Some metrics are scaled by 


\section{Type 2 diabetes}

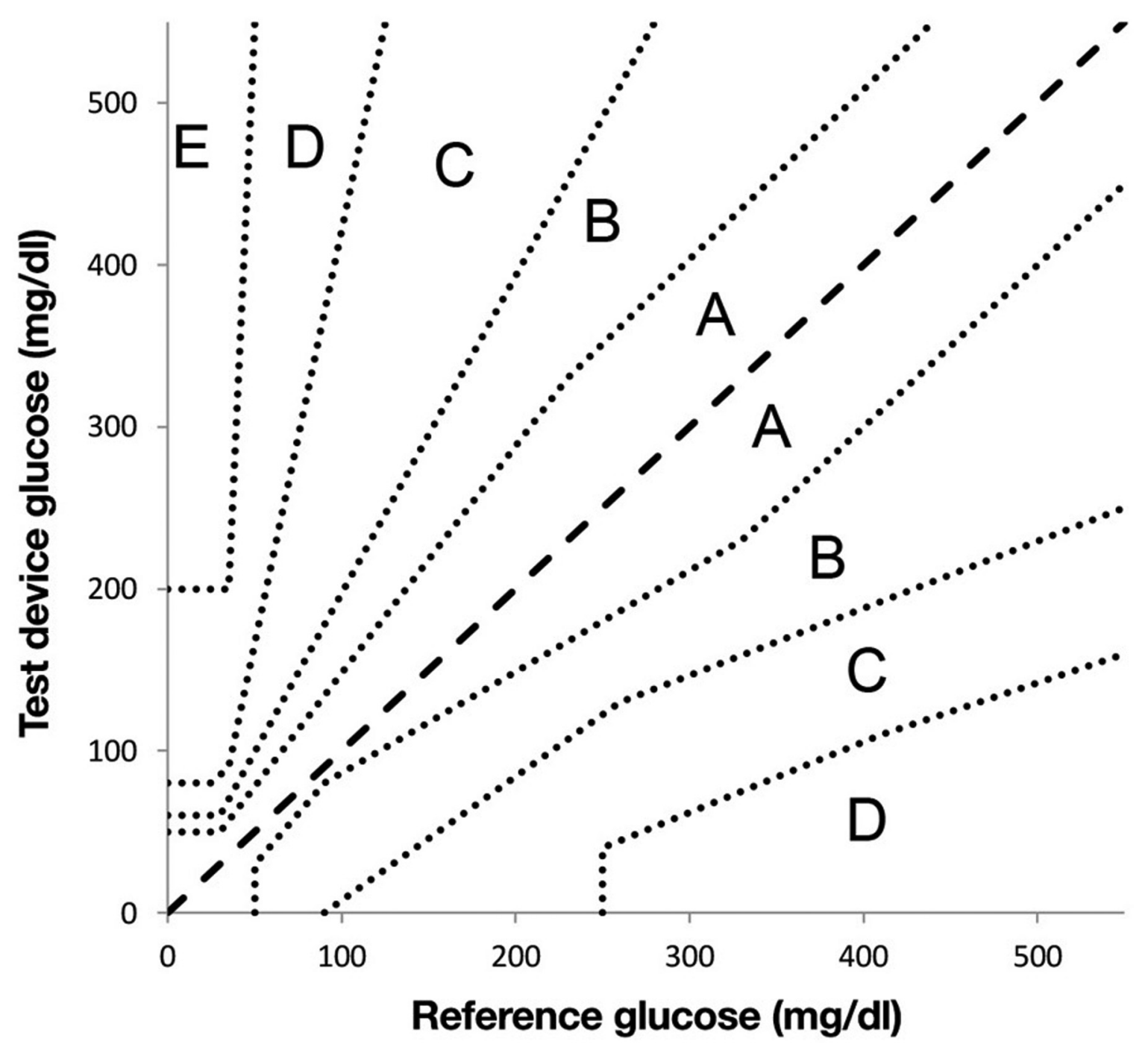

Fig. 4 Parkes error grid. This grid assigns an error severity level from A to $E$ (E high) based on the actual glucose level and the glucose level that is measured. We used forecasts in place of measurements. Image courtesy: Pfützner et al. ${ }^{15}$

100 (“\%”) or 10,000 to make them more readable. We do not have a gold standard measurement of utility, so we instead studied correlations among our metrics. We used pairwise linear correlation using the Pearson product-moment correlation coefficient between each pair of metrics.

We also performed a factor analysis using the "fa" function in the $\mathrm{R}$ statistical programming language (package "psych"). We used ordinary (unweighted) least squares to find the minimum residual (minres) solution, specifying one to five factors.

\section{Results}

-Table 4 shows the results, with some rows missing where the method did not converge. The patients in - Fig. 2 are bolded and marked with footnotes. All metrics are worse (higher implies more error) for -Fig. 2B compared with - Fig. 2A, other than the two metrics that were 0 in both. Of the two patients with less error, patients 2 and 4, we note that patient 4 was young and had a trauma-induced subdural hematoma and shorter length of stay, and patient 2 had an intraventricular hemorrhage but otherwise did not stand out as healthy. Those with subarachnoid hemorrhages tended to have higher errors.

The correlation among the metrics is shown in - Fig. 5. In general, the RMS error of the raw or transformed glucose, $g$, and Parkes errors correlated fairly well with each other, but the treatment-based metrics were less well correlated with those. The factor analysis in - Table 5 revealed more detail. The one-factor model reiterates the correlation result, that RMS error of the raw or $g$ and Parkes errors carry the most variance. The two-factor model appears to split between metrics that emphasize low versus high glucose errors, with RMS of $g$, the peak difference in low glucose, average cost, 


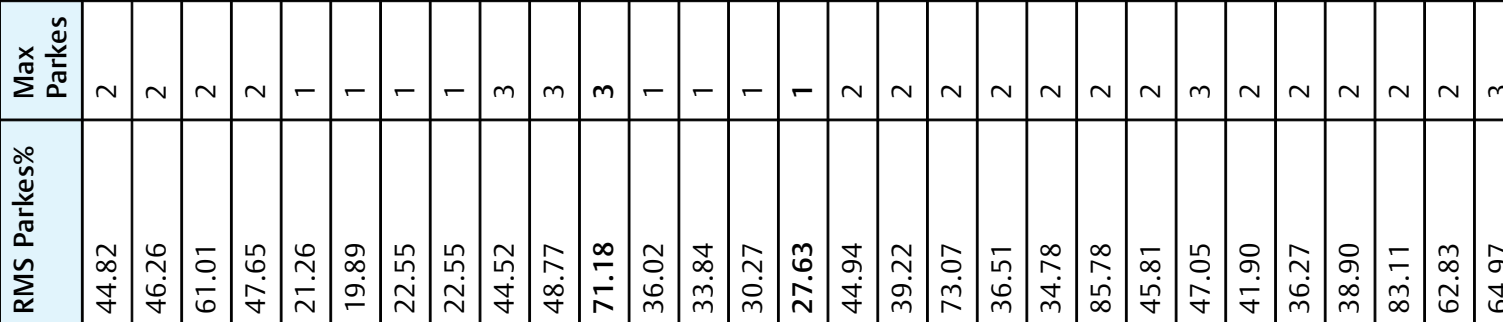

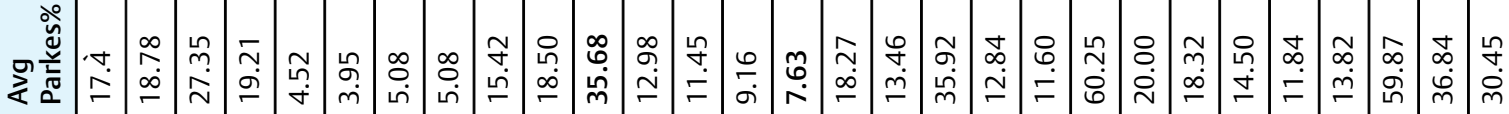

言

끙

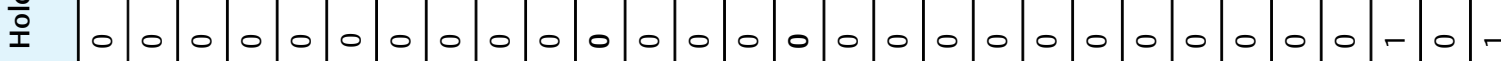

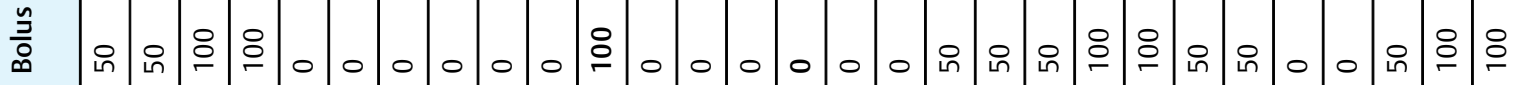

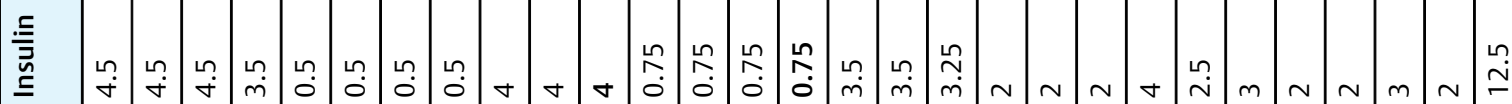
ঃ

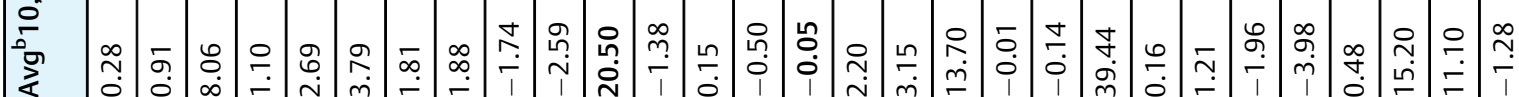
을

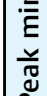

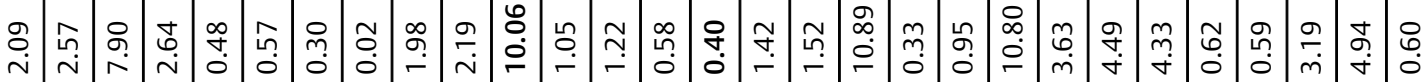

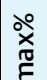

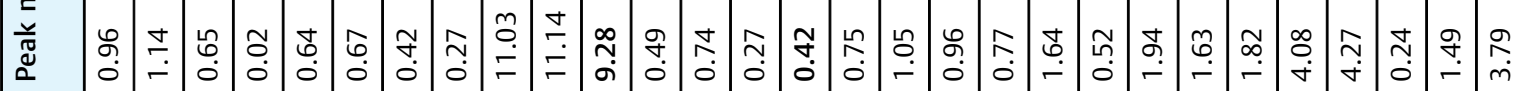
ญ̊ำ

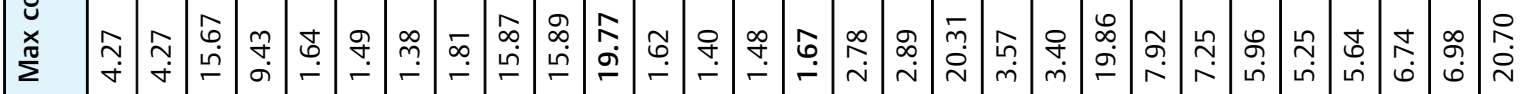

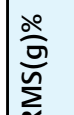

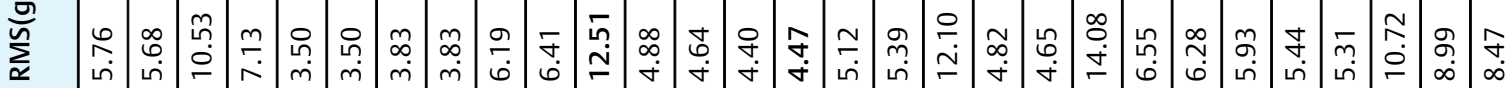
そ

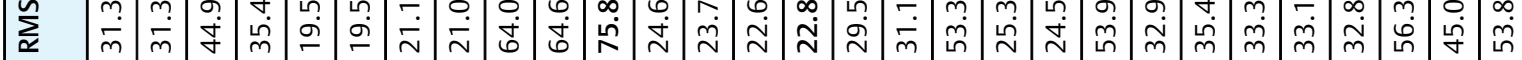

\begin{tabular}{|c|c|c|c|c|c|c|c|c|c|c|c|c|c|c|c|c|c|c|c|c|c|c|c|c|c|c|c|}
\hline $\begin{array}{l}\bar{d} \\
\overline{0} \\
\Sigma\end{array}$ & 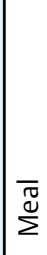 & 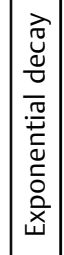 & 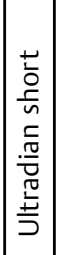 & 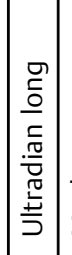 & $\mid$\begin{tabular}{|l|}
$\frac{\pi}{\tilde{J}}$ \\
$\frac{\pi}{2}$
\end{tabular} & 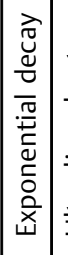 & 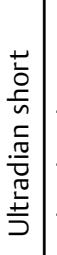 & 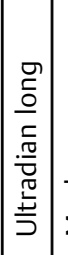 & $\frac{}{\bar{\sigma}}$ & 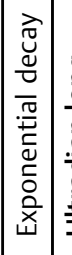 & 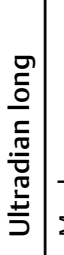 & 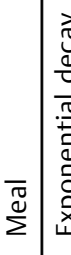 & 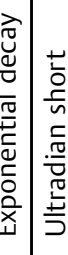 & 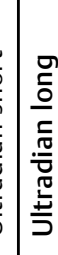 & 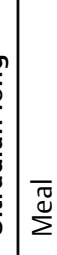 & 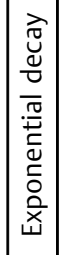 & 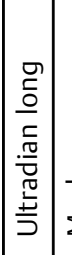 & 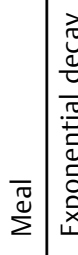 & 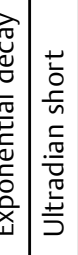 & 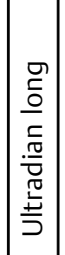 & $\mid$ & 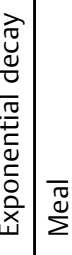 & 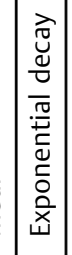 & 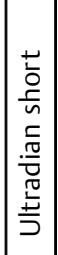 & 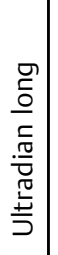 & 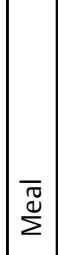 & ำ \\
\hline in & - & - & - & - & $\sim$ & $\sim$ & $\sim$ & $\sim$ & $m$ & $m$ & m & $\nabla$ & $\nabla \mid \nabla$ & $\stackrel{\sigma}{\sim}$ & ᄂn & Ln & in & \begin{tabular}{l|l}
0 & 0 \\
\end{tabular} & \begin{tabular}{l|l}
0 & 0 \\
\end{tabular} & 6 & I & \begin{tabular}{l|l}
$n$ & $\infty$ \\
\end{tabular} & $\infty$ & $\infty$ & $\infty$ & 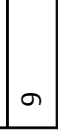 & $\overline{ }$ \\
\hline
\end{tabular}




\begin{tabular}{|c|c|c|c|c|c|c|c|c|c|c|c|c|c|}
\hline & RMS & RMS(g) & Maxcost & Peakmax & Peakmin & CostAvg & Insulin & Bolus & Hold & Notify & AvgPark & RMSPark & MaxPark \\
\hline RMS & 1.00 & 0.79 & 0.88 & 0.70 & 0.64 & 0.48 & 0.53 & 0.47 & 0.33 & 0.36 & 0.71 & 0.81 & 0.78 \\
\hline RMS(g) & 0.79 & 1.00 & 0.82 & 0.17 & 0.90 & 0.82 & 0.38 & 0.71 & 0.29 & 0.17 & 0.92 & 0.95 & 0.49 \\
\hline Maxcost & 0.88 & 0.82 & 1.00 & 0.53 & 0.73 & 0.51 & 0.62 & 0.59 & 0.26 & 0.27 & 0.65 & 0.78 & 0.71 \\
\hline Peakmax & 0.70 & 0.17 & 0.53 & 1.00 & 0.11 & -0.07 & 0.32 & -0.05 & -0.01 & 0.24 & 0.05 & 0.18 & 0.68 \\
\hline Peakmin & 0.64 & 0.90 & 0.73 & 0.11 & 1.00 & 0.78 & 0.16 & 0.64 & -0.08 & 0.10 & 0.72 & 0.78 & 0.38 \\
\hline CostAvg & 0.48 & 0.82 & 0.51 & -0.07 & 0.78 & 1.00 & -0.04 & 0.49 & 0.09 & -0.10 & 0.80 & 0.72 & 0.12 \\
\hline Insulin & 0.53 & 0.38 & 0.62 & 0.32 & 0.16 & -0.04 & 1.00 & 0.52 & 0.59 & 0.42 & 0.35 & 0.50 & 0.69 \\
\hline Bolus & 0.47 & 0.71 & 0.59 & -0.05 & 0.64 & 0.49 & 0.52 & 1.00 & 0.25 & 0.25 & 0.64 & 0.70 & 0.46 \\
\hline Hold & 0.33 & 0.29 & 0.26 & -0.01 & -0.08 & 0.09 & 0.59 & 0.25 & 1.00 & 0.32 & 0.49 & 0.45 & 0.25 \\
\hline Notify & 0.36 & 0.17 & 0.27 & 0.24 & 0.10 & -0.10 & 0.42 & 0.25 & 0.32 & 1.00 & 0.27 & 0.34 & 0.55 \\
\hline AvgPark & 0.71 & 0.92 & 0.65 & 0.05 & 0.72 & 0.80 & 0.35 & 0.64 & 0.49 & 0.27 & 1.00 & 0.96 & 0.43 \\
\hline RMSPark & 0.81 & 0.95 & 0.78 & 0.18 & 0.78 & 0.72 & 0.50 & 0.70 & 0.45 & 0.34 & 0.96 & 1.00 & 0.59 \\
\hline \multirow[t]{2}{*}{ MaxPark } & 0.78 & 0.49 & 0.71 & 0.68 & 0.38 & 0.12 & 0.69 & 0.46 & 0.25 & 0.55 & 0.43 & 0.59 & 1.00 \\
\hline & 0.65 & 0.65 & 0.64 & 0.30 & 0.53 & 0.43 & 0.46 & 0.51 & 0.32 & 0.32 & 0.61 & 0.67 & 0.55 \\
\hline
\end{tabular}

Fig. 5 Correlation among the metrics. Pearson correlation coefficient among the metrics in $=$ Table 2, colored on a scale from strong correlation (near 1 ) as green and poor or inverse correlation ( 0 and below) as red.

and average and RMS Parkes error grouped for low values and peak of the difference in high glucose, RMS error of raw glucose, and maximum Parkes error in the high group. A third factor adds the treatment metrics, insulin change, and hold insulin, as its own factor. Additional factors separate insulin change from insulin hold and pull in notification of the clinician.

\section{Discussion}

Our results can be seen from two points of view. The first point of view is related to utility. Using the treatment guideline ( - Fig. 3 ) as a surrogate for impact on clinical care, we find that all of its metrics (insulin, bolus, hold, notify) have only mediocre correlation with either the common metric, RMS error in raw glucose, or even RMS error of glucose that has been transformed to better track impact. Looking at the factors in -Table 5, the guidelinebased measurements generally have significant loadings in their own factors separate from the RMS metrics. That is, they appear to deliver different information. Therefore, at least in this domain, commonly used metrics may not in fact correlate well with effects on clinical care.

The Parkes error grid metrics have better correlation with the RMS glucose error metrics, which is not surprising because it is an algorithm based on differences in glucose measurement, rescaled roughly by using five categories, A to E. In the factor analysis, when the model is given enough factors, the average Parkes and RMS Parkes metrics remain tightly linked to the RMS error of the transformed glucose and not to the guideline-based metrics. Therefore, the Parkes error grid, which is intended to show the importance of differences in glucose, may not be a good indicator of effects on clinical care.

The second point of view is related to explaining the variance between cases: how can we best separate cases without specifically worrying about effects on clinical care. Most of the metrics appeared to reflect gross features in the time series, such as comparing the metrics for the cases shown in -Fig. 2A and B. Many of the metrics were well correlated. They grouped in a reasonable way, with the largest separation being in whether the errors appeared to be more on the low-glucose side or high-glucose side. The changes in treatment explained less variance than the more basic changes in glucose level, and they appeared to be poorly correlated with those basic changes, implying-as noted above-that they may supply useful orthogonal information. It appears that the simplest approach, RMS of difference in raw glucose, did correlate with the others, but that the transformed glucose, g, explained more variance. The combination of RMS of g, peak of the difference in high glucose, and insulin change may adequately cover the variance.

Our main limitation is that putting an algorithm like this into actual clinical practice and measuring differences in outcomes is an enormous undertaking and was out of scope for this study. Nevertheless, we believe that the actual guideline used in practice where the data were generated should cast a reasonable light on projected impact on the process of care. Second, our study was limited to nine patients from the neurological intensive care unit, and although that was sufficient to estimate the factor model and correlations, it limits the representativeness of our sample. We believe that our main messages-that is it important to explicitly evaluate evaluation metrics, that several glucose-related metrics can be enumerated, and that correlation and factor analysis can be used to assess the metrics in the absence of a gold standard-still hold and acknowledge that it would be useful to expand the clinical area beyond the neurological intensive care unit. Third, we assessed only one clinical area-glucose management-but it is a common and important one, and demonstration of a mismatch between common metrics and likely clinical care impact here at least raises the question for other areas. Fourth, we chose a particular insulin protocol for this study, shown in -Fig. 3, but protocols vary ${ }^{16}$ and could lead to different results. Fifth, we focused on the glucose point estimate, but the predicted bounds around the estimate may be more important (e.g., the likelihood of severe hypoglycemia); the bounds would be worthy of further study.

In conclusion, our results indicate that we need to be careful before we assume that commonly used metrics like RMS error in raw glucose or even metrics like the Parkes error 
grid that are designed to measure importance of differences will correlate well with actual effect on clinical care processes. A combination of metrics appeared to explain the most variance between cases. As prediction algorithms move into practice, it will be important to measure actual effects.

Table 5 Factor analysis

\begin{tabular}{|c|c|c|c|c|c|}
\hline Metric & Factor 1 & Factor 2 & Factor 3 & Factor 4 & Factor 5 \\
\hline \multicolumn{6}{|l|}{ One-factor model } \\
\hline RMS & 0.81 & & & & \\
\hline RMS(g)\% & 0.96 & & & & \\
\hline Max cost $\%$ & 0.78 & & & & \\
\hline Peak max\% & 0.18 & & & & \\
\hline Peak min\% & 0.79 & & & & \\
\hline Avg ${ }^{*} 10,000$ & 0.73 & & & & \\
\hline Insulin & 0.49 & & & & \\
\hline Bolus & 0.7 & & & & \\
\hline Hold & 0.44 & & & & \\
\hline Notify & 0.33 & & & & \\
\hline Avg Parkes\% & 0.96 & & & & \\
\hline RMS Parkes\% & 1 & & & & \\
\hline Max Parkes & 0.59 & & & & \\
\hline \multicolumn{6}{|l|}{ Two-factor model } \\
\hline RMS & 0.54 & 0.68 & & & \\
\hline RMS(g)\% & 0.98 & 0.06 & & & \\
\hline Max cost $\%$ & 0.65 & 0.46 & & & \\
\hline Peak max\% & -0.21 & 1 & & & \\
\hline Peak min\% & 0.91 & -0.05 & & & \\
\hline Avg*10,000 & 0.91 & -0.24 & & & \\
\hline Insulin & 0.22 & 0.42 & & & \\
\hline Bolus & 0.76 & -0.12 & & & \\
\hline Hold & 0.25 & 0.15 & & & \\
\hline Notify & 0.04 & 0.38 & & & \\
\hline Avg Parkes\% & 0.92 & 0 & & & \\
\hline RMS Parkes $\%$ & 0.91 & 0.13 & & & \\
\hline Max Parkes & 0.23 & 0.71 & & & \\
\hline \multicolumn{6}{|l|}{ Three-factor model } \\
\hline RMS & 0.51 & 0.66 & 0.15 & & \\
\hline RMS(g)\% & 0.95 & 0.07 & 0.06 & & \\
\hline Max cost $\%$ & 0.65 & 0.49 & 0 & & \\
\hline Peak max\% & -0.16 & 1.03 & -0.09 & & \\
\hline Peak min\% & 1 & 0.05 & -0.3 & & \\
\hline Avg $\$ 10,000$ & 0.93 & -0.18 & -0.12 & & \\
\hline Insulin & 0.07 & 0.3 & 0.53 & & \\
\hline Bolus & 0.7 & -0.12 & 0.15 & & \\
\hline Hold & -0.01 & -0.06 & 0.85 & & \\
\hline Notify & -0.08 & 0.24 & 0.49 & & \\
\hline Avg Parkes $\%$ & 0.81 & -0.07 & 0.38 & & \\
\hline RMS Parkes $\%$ & 0.81 & 0.07 & 0.33 & & \\
\hline Max Parkes & 0.17 & 0.66 & 0.27 & & \\
\hline \multicolumn{6}{|l|}{ Four-factor model } \\
\hline RMS & 0.55 & 0.64 & 0.12 & 0.05 & \\
\hline RMS (g)\% & 0.92 & 0.06 & 0.14 & -0.08 & \\
\hline Max cost $\%$ & 0.49 & 0.37 & 0.42 & -0.23 & \\
\hline Peak max\% & -0.12 & 1.03 & -0.06 & -0.03 & \\
\hline Peak min\% & 0.85 & 0.01 & 0.08 & -0.4 & \\
\hline $\mathrm{Avg}^{*} 10,000$ & 0.99 & -0.07 & -0.29 & -0.04 & \\
\hline
\end{tabular}

\begin{tabular}{|l|l|l|l|l|l|}
\hline Insulin & -0.1 & 0.08 & 0.93 & 0.14 & \\
\hline Bolus & 0.53 & -0.24 & 0.52 & -0.11 & \\
\hline Hold & 0.18 & -0.08 & 0.36 & 0.64 & \\
\hline Notify & 0.02 & 0.2 & 0.27 & 0.35 & \\
\hline Avg Parkes\% & 0.96 & 0 & -0.01 & 0.31 & \\
\hline RMS Parkes\% & 0.86 & 0.07 & 0.19 & 0.18 & \\
\hline Max Parkes & 0.13 & 0.57 & 0.42 & 0.09 & \\
\hline Five-factor model & & & & & \\
\hline RMS & 0.52 & 0.62 & 0.09 & 0.1 & 0.11 \\
\hline RMS(g)\% & 0.89 & 0.07 & 0.19 & 0 & 0.03 \\
\hline Max cost\% & 0.46 & 0.41 & 0.49 & -0.08 & 0.04 \\
\hline Peak max\% & -0.12 & 1.01 & -0.04 & 0.03 & -0.04 \\
\hline Peak min\% & 0.85 & 0 & 0.24 & 0.02 & -0.39 \\
\hline Avg*10,000 & 1 & -0.03 & -0.15 & -0.22 & 0 \\
\hline Insulin & -0.16 & 0.08 & 0.66 & 0.25 & 0.39 \\
\hline Bolus & 0.5 & -0.25 & 0.43 & 0.2 & -0.01 \\
\hline Hold & 0.1 & -0.04 & 0.1 & 0 & 0.89 \\
\hline Notify & -0.01 & 0.03 & -0.07 & 0.72 & 0.01 \\
\hline Avg Parkes\% & 0.92 & -0.03 & -0.13 & 0.18 & 0.25 \\
\hline RMS Parkes\% & 0.82 & 0.03 & 0.07 & 0.26 & 0.15 \\
\hline Max Parkes & 0.1 & 0.46 & 0.2 & 0.54 & -0.05 \\
\hline
\end{tabular}

Note: Factor loadings reshown. Loadings over 0.8 are green, and loadings 0.4 to 0.8 are yellow. Factors are sorted left to right by proportion of variance explained.

Authors' Contributions

All authors made substantial contributions to the conception and design of the work; drafted the work or revised it critically for important intellectual content; had final approval of the version to be published; and agreed to be accountable for all aspects of the work in ensuring that questions related to the accuracy or integrity of any part of the work are appropriately investigated and resolved.

Funding

This work was funded by grants from the National Institutes of Health R01 LM006910 "Discovering and applying knowledge in clinical databases" and R01 LM012734 "Mechanistic machine learning."

Conflict of Interest

None declared.

\section{References}

1 Albers DJ, Levine ME, Stuart A, Mamykina L, Gluckman B, Hripcsak G. Mechanistic machine learning: how data assimilation leverages physiologic knowledge using Bayesian inference to forecast the future, infer the present, and phenotype. J Am Med Inform Assoc 2018;25(10):1392-1401

2 Law K, Stuart A, Zygalakis K. Data Assimilation. New York, NY: Springer; 2015

3 Sturis J, Polonsky KS, Mosekilde E, Van Cauter E. Computer model for mechanisms underlying ultradian oscillations of insulin and glucose. Am J Physiol 1991;260(5 Pt 1):E801-E809

4 Dalla Man C, Rizza RA, Cobelli C. Meal simulation model of the glucose-insulin system. IEEE Trans Biomed Eng 2007;54(10): 1740-1749 
5 Albers DJ, Levine M, Gluckman B, Ginsberg H, Hripcsak G, Mamykina $\mathrm{L}$. Personalized glucose forecasting for type 2 diabetes using data assimilation. PLOS Comput Biol 2017;13(04):e1005232

6 Albers DJ, Elhadad N, Claassen J, Perotte R, Goldstein A, Hripcsak G. Estimating summary statistics for electronic health record laboratory data for use in high-throughput phenotyping algorithms. J Biomed Inform 2018;78:87-101

7 Albers DJ, Blancquart P-A, Levine ME, Seylabi EE, Stuart A. Ensemble Kalman methods with constraints. Inverse Probl 2019;35(09):095007

8 Hripcsak G, Albers DJ, Perotte A. Parameterizing time in electronic health record studies. J Am Med Inform Assoc 2015;22(04): 794-804

9 Albers DJ, Levine ME, Sirlanci M, Stuart AM. A simple modeling framework for prediction in the human glucose-insulin system. arXiv preprint arXiv: 1910.14193

10 Xiao C, Choi E, Sun J. Opportunities and challenges in developing deep learning models using electronic health records data: a systematic review.J Am Med Inform Assoc 2018;25(10):1419-1428
11 Jolliffe IT, Stephenson DB, eds. Forecast Verification: A Practitioner's Guide in Atmospheric Science, 2nd ed. Chichester, UK: John Wiley \& Sons; 2012

12 Malouf R, Brust JCM. Hypoglycemia: causes, neurological manifestations, and outcome. Ann Neurol 1985;17(05): 421-430

13 Adeyinka A, Kondamudi NP. Hyperosmolar Hyperglycemic Nonketotic Coma (HHNC, Hyperosmolar Hyperglycemic Nonketotic Syndrome). StatPearls. Treasure Island, FL: StatPearls Publishing; 2019

14 Parkes JL, Slatin SL, Pardo S, Ginsberg BH. A new consensus error grid to evaluate the clinical significance of inaccuracies in the measurement of blood glucose. Diabetes Care 2000;23(08): 1143-1148

15 Pfützner A, Klonoff DC, Pardo S, Parkes JL. Technical aspects of the Parkes error grid. J Diabetes Sci Technol 2013;7(05):1275-1281

16 Wilson M, Weinreb J, Hoo GW. Intensive insulin therapy in critical care: a review of 12 protocols. Diabetes Care 2007;30(04): 1005-1011 